\title{
The Effect of the Extract of Immature Bean Seeds on the Growth of Coleoptile and Leaf of Rice Plant
}

\author{
by Yutaka MuraKami*
}

村上 浩*：イネの子葉鞘と葉の生長に及ぼす未熟な菜豆の抽出物の影響

Received September 28, 1957

The hormone relationships in the growth of cereal leaves are less understood than those in the coleoptile growth. In the previous papers ${ }^{1), 2}$, it was demonstrated that the sucrose-induced growth in length of the leaf sheath sections isolated from young cereal leaves was greatly inhibited by auxin such as indoleacetic acid (IAA) but stimulated by gibberellin. This IAA-inhibition was tentatively interpreted in terms of the so-called two-factor scheme proposed by Went3). If this scheme is valid, then it will be expected that the growth of the basal regions of green leaves should not be affected by auxin. On the other hand, various growth substances other than IAA have now been found in several kinds of plant materials. Thus a principle or principles contained in the extract of immature bean seeds as reported by Mitche1l, Skaggs, and Anderson ${ }^{4}$ is an example. They observed that by the treatment with this extract the main axis of bush beans extended to result in the formation of very long internodes as in climbing beans. The writer found that similar responses were also observed if gibberellin was applied to bush beans. Hence, it seemed interesting to examine whether or not the growth of the intact rice seedlings is modified by the application. of the above extracts. Such a study might give a clue to the elucidation of the hormone relationships in. the growth of cereal leaves.

\section{Methods}

The rice seed used in these experiments was a race called "Aichi-Asahi". Seeds were soaked for 20 minutes in a $0.1 \%$ solution of "Uspulun". They were then washed thoroughly and allowed to germinate at about $28^{\circ} \mathrm{C}$. When the emerging coleoptiles attained about $1 \mathrm{~mm}$ in length, the uniform seedlings were selected and used for experiments.

The extraction of immature bean seeds was carried out in a manner described by Mitchell et $\mathrm{al}^{4}$. Approximately $30 \mathrm{~g}$ of immature bean. seeds taken from market pods that varied from 10 to $15 \mathrm{~cm}$ in length were extracted with moist

\footnotetext{
* National Institute of Agricultural Sciences, Nishigahara, Tokyo. 農業技術研究所
} 
ether at room temperature for 20 hours. The ether extract was evaporated and the residue was dissolved in $0.5 \mathrm{ml}$ of $0.01 \mathrm{~N}$ sodium carbonate for paper chromatographic studies.

The extract so obtained was spotted on the starting line of a sheet of Tôyô No. 50 filter paper $(25 \mathrm{~cm} \times 35 \mathrm{~cm})$, in 5 small spots about $2 \mathrm{~cm}$ apart (Fig. $1, \mathrm{~A} \sim \mathrm{C}$ ). The chromatogram was developed in the ascending manner in the solvent mixture of isopropyl alcohol/water/ammonia $(D=0.88)(10: 1: 1)$ at about $25^{\circ} \mathrm{C}$ for 16 hours, by which time the solvent front travelled about $28 \mathrm{~cm}$. A mixture of IAA, ethyl indoleacetate (EIA), and indoleacetonitrile (IAN) was chormatographed simultaneously. (Fig. 1, D). The paper was then dried and cut lengthwise into 4 strips. These will be referred to as strip A, strip B, etc. Strips A and B were again divided transversely into 14 zones at $2 \mathrm{~cm}$ intervals from the starting line. (see Fig. 1). Each chromatogram piece of strip A was placed in a wide-mouth bottle $3 \mathrm{~cm}$ in diameter and $8 \mathrm{~cm}$ in height. To each bottle 1 $\mathrm{ml}$ distilled water was added, and 10 rice seedlings were planted in it. All bottles were placed in a large Petri dish which was kept in the glasshouse at about $28^{\circ} \mathrm{C}$. The plants were grown under normal daylight conditions and were supplied with $0.5 \mathrm{ml}$ distilled water every 2 days. The length of coleoptile and that of the first and second leaf were measured after 2 days and 7 days, respectively. At that time each leaf had practically completed its growth.

Strip B was used for the Avena straight-growth

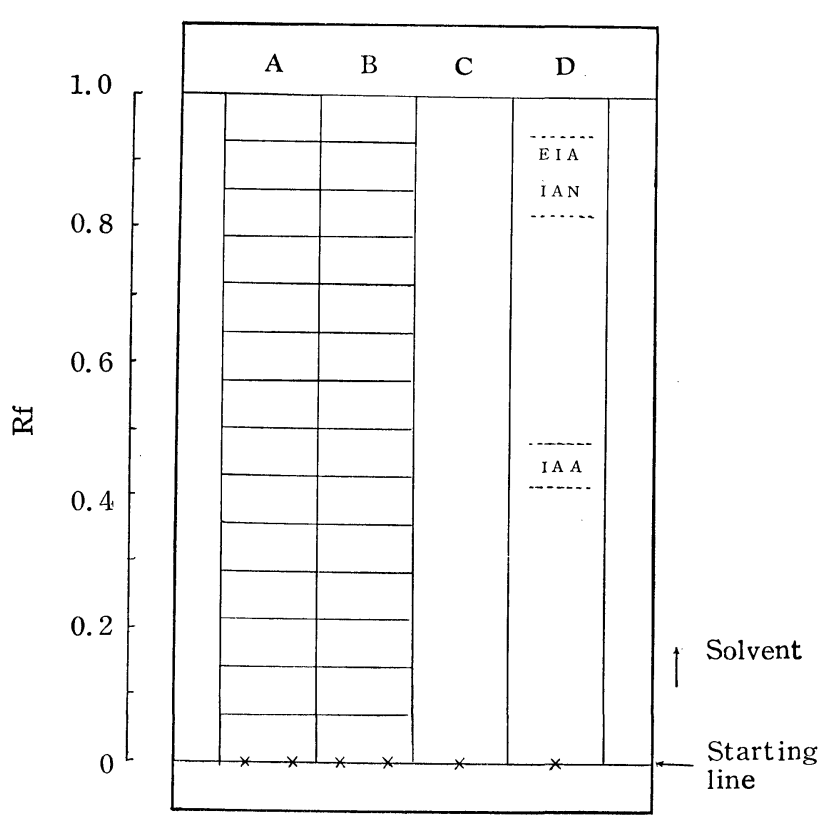

Fig. 1. Division of paper chromatogram of the ether extract of immature bean seeds.

The chromatogram was developed in isopropyl alcohol /water/ammonia $(10: 1: 1)$. Strip A was used for the determination of activity in the growth of rice seedlings, strip B for Avena straight-growth assay, strip C for spraying with p-dimethylaminobenzaldehyde, strip $D$ for determinations of standard spots of IAA, EIA, and IAN. assay of growth substances. Each paper piece was immersed in. $2 \mathrm{ml}$ distilled water in a wide-mouth bottle, and twelve $3.1 \mathrm{~mm}$ sections obtained from $2 \mathrm{~cm}$ oat coleoptiles were floated on the test solution. The bottles were placed in a water-saturated atmosphere at $25^{\circ} \mathrm{C}$. The lengths of coleoptiles were measured after incubation for 18 hours. 
The positions on the paper chromatogram of known indole compounds were detected on paper strip $\mathrm{D}$ by spraying with p-dimethylaminobenzaldehyde $(2 \mathrm{~g}$ in $20 \mathrm{ml} \mathrm{HCI}$ (S.G. 1.16) $+80 \mathrm{ml}$ ethyl alcohol). Under these conditions IAA had Rf 0.45 , EIA Rf 0.9 , and IAN Rf 0.85 .

IAA was a commercial product. The method of Jackson ${ }^{5)}$ was used to prepare EIA. IAN was prepared from gramine as described by Henbest, Jones and Smith ${ }^{6}$. Gibberellin A mixture was kindly provided by Prof. Y. Sumiki.

\section{Results and Discussion}

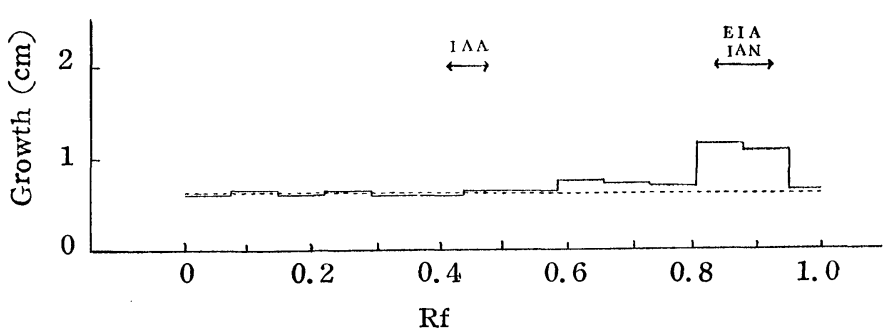

Fig. 2. Activity in the growth of intact rice coleoptile, shown as histogram, of pieces cut from paper strip A.

Measurements were made after 2 days. Broken line represents the growth of control.

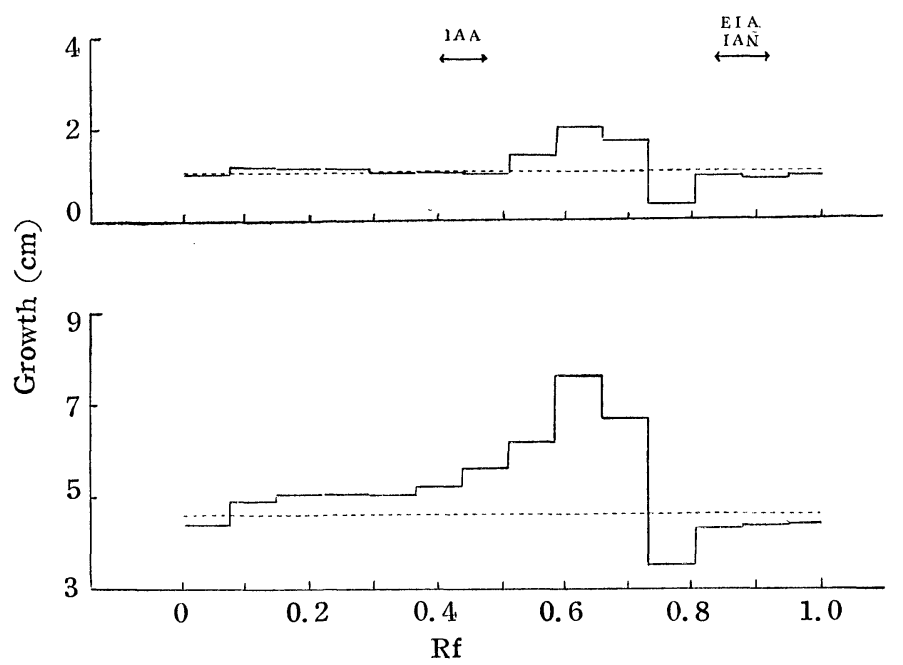

Fig. 3. Activities in the growth of foliage leaves of rice plants, shown as histograms, of pieces cut from paper strip A.

The upper histogram shows the growth of first leaf and the lower one shows that of second leaf. Measurements were made after 7 days. Broken lines represent the growth of controls.

The results of the experiments with paper strip A have been represented as histograms of the lengths of the coleoptile (Fig. 2), the first leaf (Fig. 3 ), and the second leaf (Fig. 3), plotted against Rf of the chromatogram. The horizontal broken lines in the figures represent the growth of controls.

In Fig. 2, where the effect of the extract on the growth of intact rice coleoptile was presented, only one growth-active zone was apparent at $\mathrm{Rf} 0.9$. This growth promotion may be due to EIA or IAN since the active region lies close to the positions of standard EIA and IAN. Unlike the histogram pattern of Fig. 2, that of Fig. 3 representing the effects of the extract on the foliage-leaf growth showed one growth inhibiting zone of Rf 0.75 and one growth promoting zone 
of $\mathrm{Rf}$ 0.65. In both figures, however, there was no peak of growth promotion corresponding to IAA. In paper strip $\mathrm{C}$ sprayed with p-dimethylaminobenzaldehyde, a pale purple colouration appeared at the region of $\mathrm{Rf} 0.9$, and no coloured spots could be detected in other parts of the strip. Therefore, it may be considered probable that the concentration in the bean extract of IAA must be very low if it is present at all.

The Avena straight-growth assay is known to be more sensitive than chromogenic detection of IAA on the chromatogram. Hence, the paper strip B was examined for the presence of IAA by the method of Avena straight-growth assay. The results are shown in Fig. 4, where two clear zones with growth activity were ap-

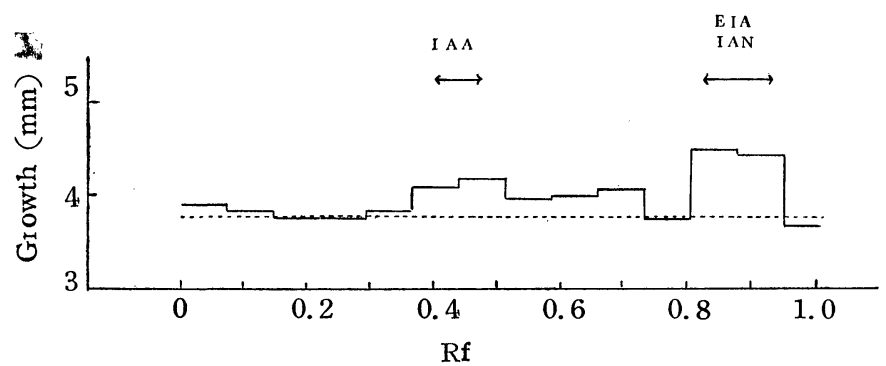

Fig. 4. Activity in Avena straight-growth assay, shown as histogram, of pieces cut from papər strip $B$. Initial length of section was $3.1 \mathrm{~mm}$. Broken line represents the growth of control.

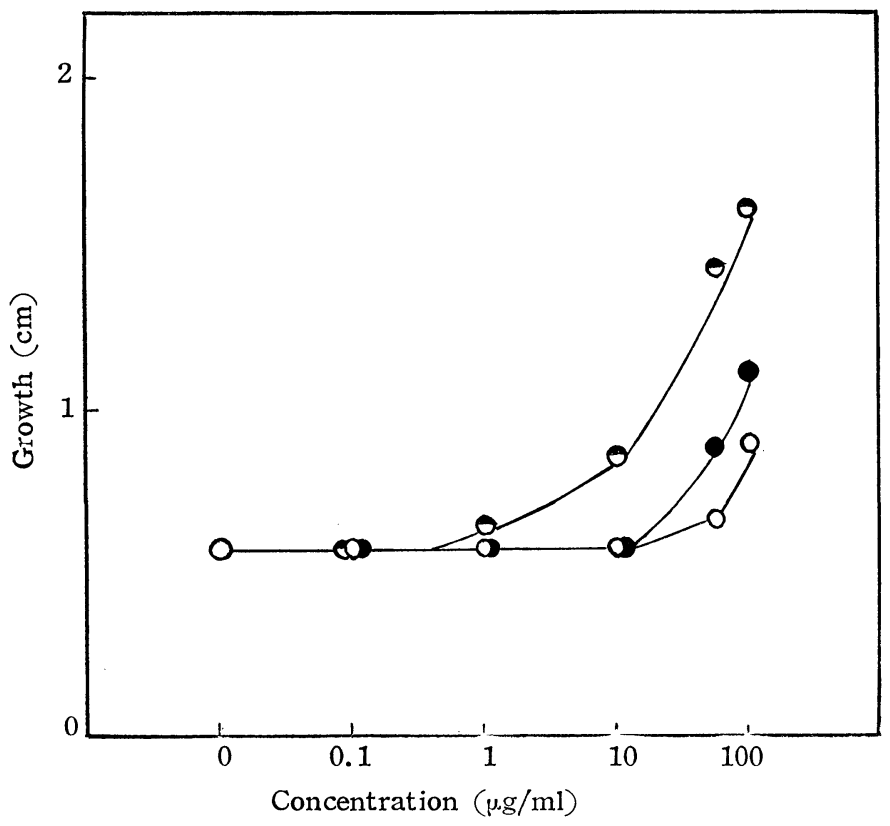

Fig. 5. Effects of various concentrations of IAA, EIA and IAN on the growth of intact rice coleoptile.

(O) IAA, (O) EIA, (O) IAN. parent. A zone of Rf 0.9 having growth-promoting activity for the Avena coleptile sections corresponded to the area marked by the stimulation of the growth of intact rice coleoptile. The other active zone of Rf 0.45, which corresponded to the position of the IAA marker spot, might be due to IAA.

IAA seemed to be less effective on the intact rice coleoptile, since its growth was not promoted by the eluate from the zone of $\mathrm{Rf}$ 0.45. Accordingly, the effect of IAA, EIA and IAN on the growth of intact rice coleoptile was compared. Ten seedlings were planted in $1 \mathrm{ml}$ of each test solution in the bottles, and the length of coleoptile was measured after 2 days. Results of the typical experiment are represented graphically in Fig. 5. The data show that EIA was the most 
active compound in promoting the intact coleoptile elongation, and that it was the only compound tested having activity at low concentrations of $1-10 \mu \mathrm{g} / \mathrm{ml}$. Also, IAN was active at concentrations above $10-50 \mu \mathrm{g} / \mathrm{ml}$. However, IAA was inactive at concentrations below $50 \mu \mathrm{g} / \mathrm{ml}$. These facts suggest that free IAA is not always favourable for the growth of the intact rice coleoptile.

If IAN was present at concentrations above $50 \mu \mathrm{g} / \mathrm{ml}$ on the region of $\mathrm{Rf} 0.9$, then one would expect to obtain an intense purple spot with p-dimethylaminobenzaldehyde. As described above, the intensity of the colour developed with this reagent on the region of $\mathrm{Rf} 0.9$ was too weak to account for the biological activity. Therefore, the compound detected on the chromotogram responsible for promoting growth of rice coleoptile may be EIA rather than IAN. For the identification of this compound a much larger scale experiment would be needed, and it will be the subject of future research.

As previously stated, the histogram pattern of foliage leaves differed from that of coleoptiles. Namely, in the former no activity could be detected on the area corresponding to EIA, and growth promotion was found at the zone with $\mathrm{Rf} 0.65$ instead. A zone just above the growth-promoting zone showed inhibition of the growth of leaves. It appears that the substance in this zone might be identical with the inhibitor- $\beta$ of Bennet-Clark and Kefford ${ }^{7}$. The failure of the colour reaction with p-dimethylaminobenzaldehyde on these regions of strip $\mathrm{C}$ suggests that indole compounds were not involved in the growth activity of the leaf.

The active principles were separated by large scale paper chromatography. From a number of chromatograms developed on a large sheet of paper, portion of the strip having growth-promoting activity was excised and eluted separately with boiling $70 \%$ ethyl alcohol. The eluate was concentrated and was applied in form of a small drop to the unfolding leaf of young bush beans. Only that eluate which promoted the growth of rice foliage leaves was able to affect the growth of bush beans, which grew like pole types by applying this eluate. The other eluates were almost inactive. Therefore, it seems likely that the effect of the extract observed by Mitchell et al. may be attributed to a factor located in the zone of Rf 0.65 with the solvent system used. Rice seedlings treated with this eluate grew taller and their appearance was similar to that of gibberellin-treated plants. Hence, for the sake of comparison, the Rf value of gibberellin A was measured with the same solvent system as employed above. The location of gibberellin A was made by bioassay of each successive region of the chromatogram with rice seedlings as test plant. A typical result is shown in Fig. 6. It is interesting that Rf of gibbere11in A, 0.6-0.7, coincides with that of the growth-promoting factor of the bean extract.

Recently, Radley8) obtained evidence for the occurrence of substances resembling gibberellic acid in the extracts of young pea shoots. On paper chromatograms, using solvent mixture of chloroform/ethyl alcohol/water/formic acid (20:4:2:1), 
an active zone was found to have the same $\mathrm{Rf}$ value as that of gibberellic acid. Very recently, Phinney, West, Ritzel and Neely ${ }^{9}$ have also reported that in a variety of higher plants including beans substances are present which are indistinguishable from gibberellins in respect of their biological activity towards dwarf mutants of maize.

In auxin studies it is frequently presumed that IAA or related indole compounds are either the dominant hormone or the only naturally occurring growth promoter in plants. Thus IAA, EIA and IAN have now been isolated in crystalline form from different plants. Auxin such as IAA has, in fact, been shown to stimulate the growth of the sections of oat coleoptiles, and the

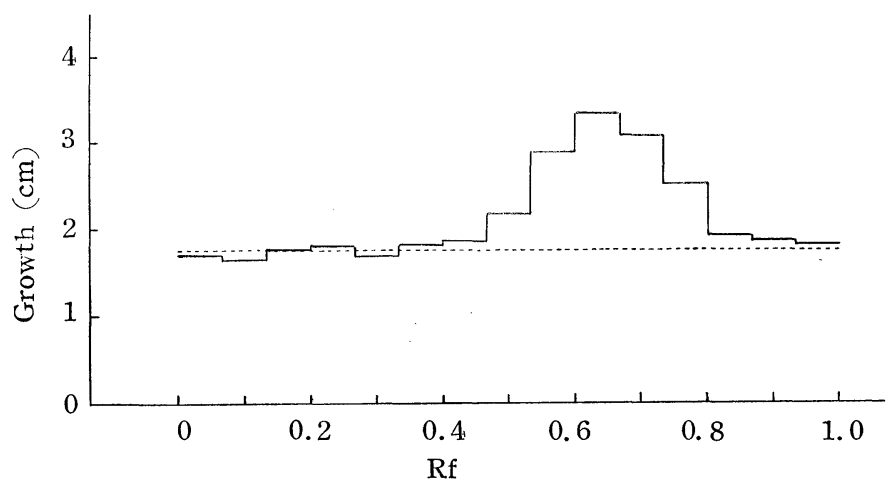

Fig. 6. Paper chromatography of gibberellin A $(25 \mu \mathrm{g})$ in ammoniacal isopropyl alcohol.

Biological assay of gibberellin A was made by measuring the length of second leaf of rice plant after 4 days. Bro. ken line represents the length of control.

"Avena test" is widely used for the identification and quantitative estimation of natural plant growth hormones. However, auxin is almost inert for the growth of intact green plants. Although nothing is known at present about the chemical nature of the active principle of the bean extract, the experimental data seem to favour the view that indole compounds might not be involved in the growth promotion of foliage leaves of rice plants in the present work.

\section{Summary}

The effect of the ether extract of immature bean seeds on the growth of rice seedlings was studied. Attempts were made to separate active principles by paper chromatography.

The growth of the intact coleoptile was promoted by the eluate from the zone of $\operatorname{Rf} 0.9$ in the solvent mixture of isopropyl alcohol/water/ammonia $(10: 1: 1)$. EIA and IAN showed similar Rf to that of the coleoptile growth-promoting substance of the bean extract. Synthetic EIA was found to be 10 times more effective than IAA on the pormotion of rice coleoptile growth.

On the other hand the growth of the intact foliage leaf was promoted only by the eluate from the zone of Rf 0.65 and inhibited by that of Rf 0.75. Gibberellin A showed similar Rf to that of the leaf growth-promoting substance of the bean extract.

The writer gratefully acknowledges the advice and helpful criticism which he 
has received from Prof. T. Miwa and Dr. T. Hayashi during the experiments and in the preparation of this report.

\section{References}

1) Hayashi, T. and Murakami, Y., J. Agr. Chem. Soc. Japan, 28, 543 (1954). 2) Murakami, Y., Bot. Mag. Tokyo 69, 258 (1956). 3) Went, F. W. and Thimann, K. V., Phytohormones, New York (1937). 4) Mitchell, J.W., Skaggs, D.P., and Anderson, W.P., Science, 114, 159 (1951). 5) Jackson, R., J. Biol. C'iem. 88, 660 (1930). 6) Henbest, H.B., Jones, E. R. H. and Smith, G. F., J. Chem. Soc. 3793 (1953). 7) Bennet-Clark, T. A. and Kefford, N.P., Nature 171, 645 (1953). 8) Radley, M., Nature, 178, 1070 (1956). 9) Phinney, B. O., West, C. A., Ritzel, M. and Neely, P. M., Proc. Nat. Acad. Sci., 43, 398 (1957). 